\title{
T Tauri stars: from mystery to magnetospheric accretion
}

\author{
Gibor Basri \\ Department of Astronomy, University of California, Berkeley, CA 94720, USA \\ email: Basri@berkeley.edu
}

\begin{abstract}
This is a selective historical overview of the progess in understanding T Tauri spectra. Originally they were understood to be very young, but the physical conditions (or even geometry) of the material on the star and in its surroundings were mysterious. The origin and meaning of the emission lines was largely unknown. Today we have a detailed consensus of what is happening near and on these newly forming stars. They are very magnetically active, and the stellar field is strong and extensive enough to control both the final accretion onto the star and the launching of outflows which solve the angular momentum problem during formation. Much of this consensus has emerged from spectral information, but much remains to be learned. I highlight some of the seminal breakthroughs that have led to the current picture. There are very complex and time-variable components to the entire physical system that constitutes a $\mathrm{T}$ Tauri star, and spectral information at various wavelengths and resolutions is crucial to making further progress.
\end{abstract}

Keywords. Accretion disks, line: formation, line: profiles, stars: magnetic fields, stars: formation, stars: activity

The T Tauri stars provide us with a detailed glimpse into the processes that led to the formation of our own Sun and planetary system. Rather than speculating on our origins, we are able to make detailed observations of the physical processes at work. While imaging is very informative in providing context and morphological information at large scales, spectroscopy provides our chance to delve right into the detailed processes and dynamics at the heart of the star formation process. The spectra of $\mathrm{T}$ Tauri stars were enigmatic right from the first observations, suggesting that these are no ordinary stars, but rather unique forces and conditions are in play. Although they share some similarities with other types of stars, a number of remarkable spectral characteristics made it clear that once we can untangle the meaning of their spectra, we will gain insight into crucial new processes in the environments and lives of stars.

Bertout (this volume) provides an excellent overview of the whole journey from a mysterious new class of objects to our current understanding of the essential nature of $\mathrm{T}$ Tauri stars (TTS). In this overview, I concentrate on the interpretation of spectra, both low and high resolution, and in the time domain. My career spans the time between confusion on the basic physical scenarios which might explain these spectra and excellent consensus that we are looking at young magnetically active stars surrounded by accretion disks, in which magnetic fields play a crucial role in simultaneous infall and outflow regions surrounding the star. I make no pretense at a comprehensive review, but give a somewhat personal tour through some of the highlights of this journey in the spectral domain. I tend to cite historical work rather than the latest results. This should be useful for workers new to the field in providing a context for current work. My basic message is the need for simultaneous diagnostics which probe different parts of the same system, and the need to include the time domain when studying these highly variable and complex systems. 


\section{Basic Characteristics of T Tauri Spectra}

\subsection{Low Resolution: Chromospheres and Accretion}

At low resolution, the basic set of optical spectra that characterize the $\mathrm{T}$ Tauri stars are illustrated in Figure 1, taken from Bertout (1989). They illustrate the basic points about TTS spectra. Firstly, it was recognized from early on (Joy 1945) that many of the emission lines can also be seen in the solar chromosphere. Dumont et al. (1973) took the earlier suggestion of Herbig and pushed the chromosphere to high optical depth (also adjusting its temperature) and computed $\mathrm{H} \alpha$ line fluxes and profiles. While the fluxes could be reproduced (albeit with arbitrary large heating requirements), the profiles had strong (unobserved) central reversals. The chromospheric hypothesis was explored further by a number of authors, and studied with more modern methodology by Calvet, Basri \& Kuhi (1984). These authors concluded that a number of TTS features could be reproduced by extremely strong chromospheres (without explaining how such strong chromospheres could be actually supported). These included line fluxes in CaII, MgII, and some iron lines, as well as some of the blue continuum veiling. There were concerns that the stronger absorption lines had bright emission cores (not observed) in such models, but NLTE effects had not been properly treated. It was clear, however, that certain spectral characteristics could never be reproduced simply by a very strong chromosphere (both strength and breadth as well as profile shape are issues). The $\mathrm{H} \alpha$ lines of the more active TTS, in particular, have to arise in a dynamic region off the star. Models which might work for $\mathrm{H} \alpha$ did great violence to other diagnostics (MgII, for example) compared with observed characterics. These conclusions were reinforced by Herbig (1985).

Nonetheless, it is actually true that TTS are extreme examples of stellar magnetic activity. Finkenzeller \& Basri (1987) provide a very graphic example of this by comparing eclipses in software for TTS (spectra divided by inactive standards) with a solar eclipse spectral image. Ultraviolet and X-ray comparisons also place many TTS at the top of a progression of increasing activity that reaches back to the Sun (eg. Calvet et al. 1985). There is a set of TTS which appear to just have strong chromospheres; they do not exhibit diagnostics of disk accretion or winds but are otherwise similar to classic TTS. These are now known as "weak-lined" TTS or WTTS. Although many make the mistake of defining them as having $\mathrm{H} \alpha$ strengths less than a certain equivalent width (typically $10 \AA$ ), one must be careful of the spectral class if using that method (see White \& Basri 2003), who suggest using profile widths to distinguish them if possible). Only the lowest spectrum in Figure 1 is from a WTTS. The first direct proof that WTTS have very strong magnetic fields came from a novel Zeeman method employed by Basri, Marcy \& Valenti (1992). These and subsequent measurements (discussed in more depth by Johns-Krull in this volume) provide one of the fundamental requirements for claiming magnetospheric accretion on TTS.

The other spectral characteristic somewhat unique to TTS which points at the presence of accretion is the so-called spectral "veiling". It was noticed very early on, even in low dispersion spectra (Figure 1), that the depth or strength of stellar absorption lines was smaller in some TTS and there are even cases in which no absorption lines are seen at all. Rydgren, Strom \& Strom (1976) give a nice summary of the evidence that a bluish continuum light superposed on a normal stellar spectrum could be the cause. They identify the Balmer continuum as in emission in the more heavily veiled cases, and suggest that an optically thin Paschen continuum is responsible for most of the rest. They were still thinking in terms of an ionized wind as the source of the continuum.

Basri \& Bertout (1989) correctly identified the veiling as due to the accretion shock from disk accretion, with a small contribution from the disk itself in the far red, increasing 


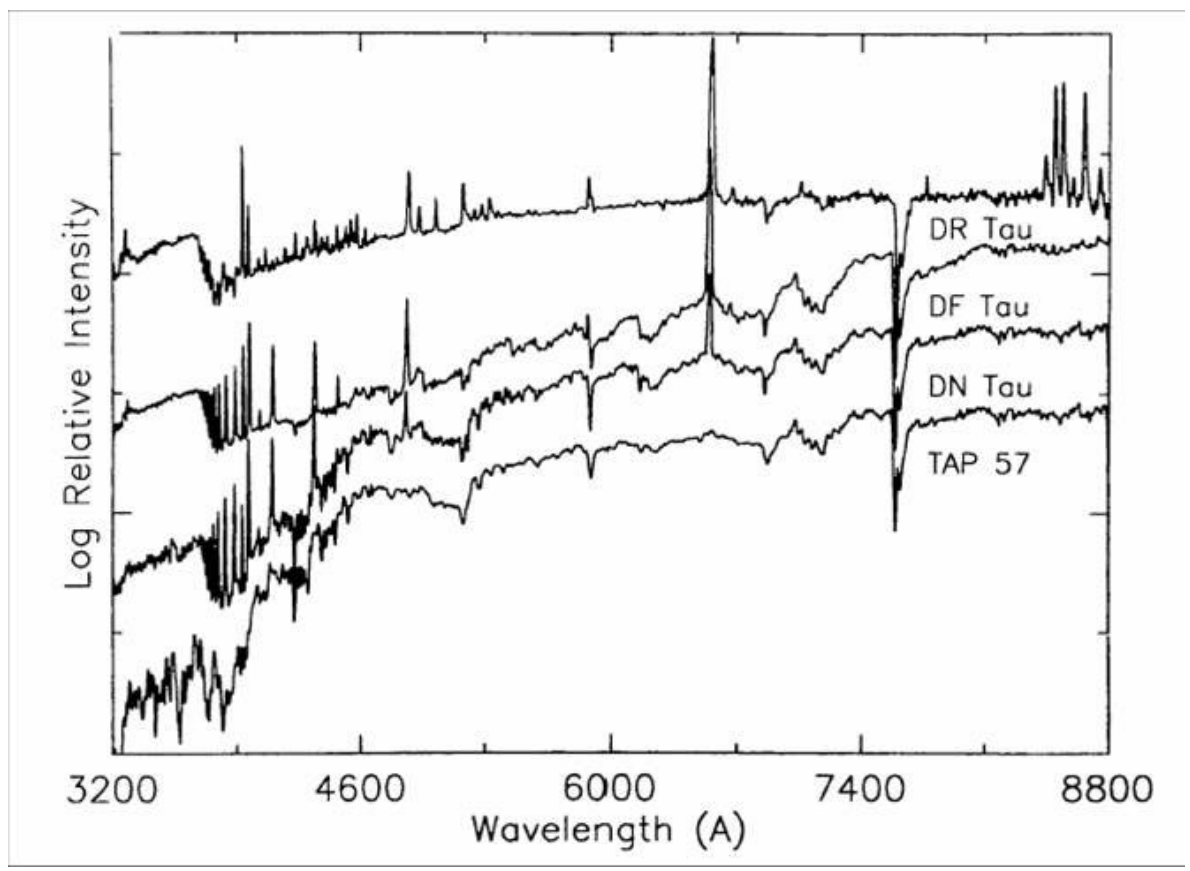

Figure 1. Low Resolution Spectra of TTS. Four sample spectra in the optical covering a range of accretion activity from none to high (moving upward). The underlying stars are all late $\mathrm{K}$ spectral type, and the bottom WTTS shows only the effects of a strong chromosphere. As accretion increases, Balmer line and continuum emission is increasingly apparent (with $\mathrm{H} \alpha$ becoming much stronger than the underlying continuum), while stellar absorption features are diluted by continuum veiling. In the upper spectrum, the Ca II infrared triplet has come strongly into emission, while the blue end is dominated by the Ca II H\&K lines and a forest of iron emission lines. No absorption features remain, and the continuum is markedly bluer throughout.

to domination in the infrared (Figure 2). They suggest that in fact this veiling continuum is one of the cleanest diagnostics for accretion rates, which has proved to be the case. The methodology and first detailed measurement of the spectral characteristics of the veiling were pioneered by Hartigan et al. (1989) and Basri \& Batalha (1990). An extensive set of accretion rates were produced by several authors, including Valenti, Basri \& Johns (1993), Hartigan, Edwards \& Ghandour (1995), and Gullbring et al. (1998). Unfortunately, the accuracy of these accretion rates is still problematic to within factors of a few, due to the complex region in which the diagnostic arises.

\subsection{High Resolution: Accretion and Outflow}

Even in the days before the discovery of bipolar molecular outflows or collimated optical jets, it was clear from line profiles that TTS have dynamic flows around them. Although the typical TTS does not exhibit a classical P Cygni profile at $\mathrm{H} \alpha$ (strong blue-shifted absorption below the continuum), many of them do show blue-shifted absorption features superposed on broad and strong emission. The broad strong emission by itself implies dynamic flows off the stellar surface, since its strength relative to the continuum is derived in part from the fact that it arises from a volume substantially larger than the photosphere. Its breadth is also thought to arise at least in part due to strong turbulent and organized flows (Doppler shifts rather than opacity broadening). The blue-shifted absorption has to arise in a cooler region (or more technically, a region with a lower source function) on the outside of the main emission region which is flowing toward the 

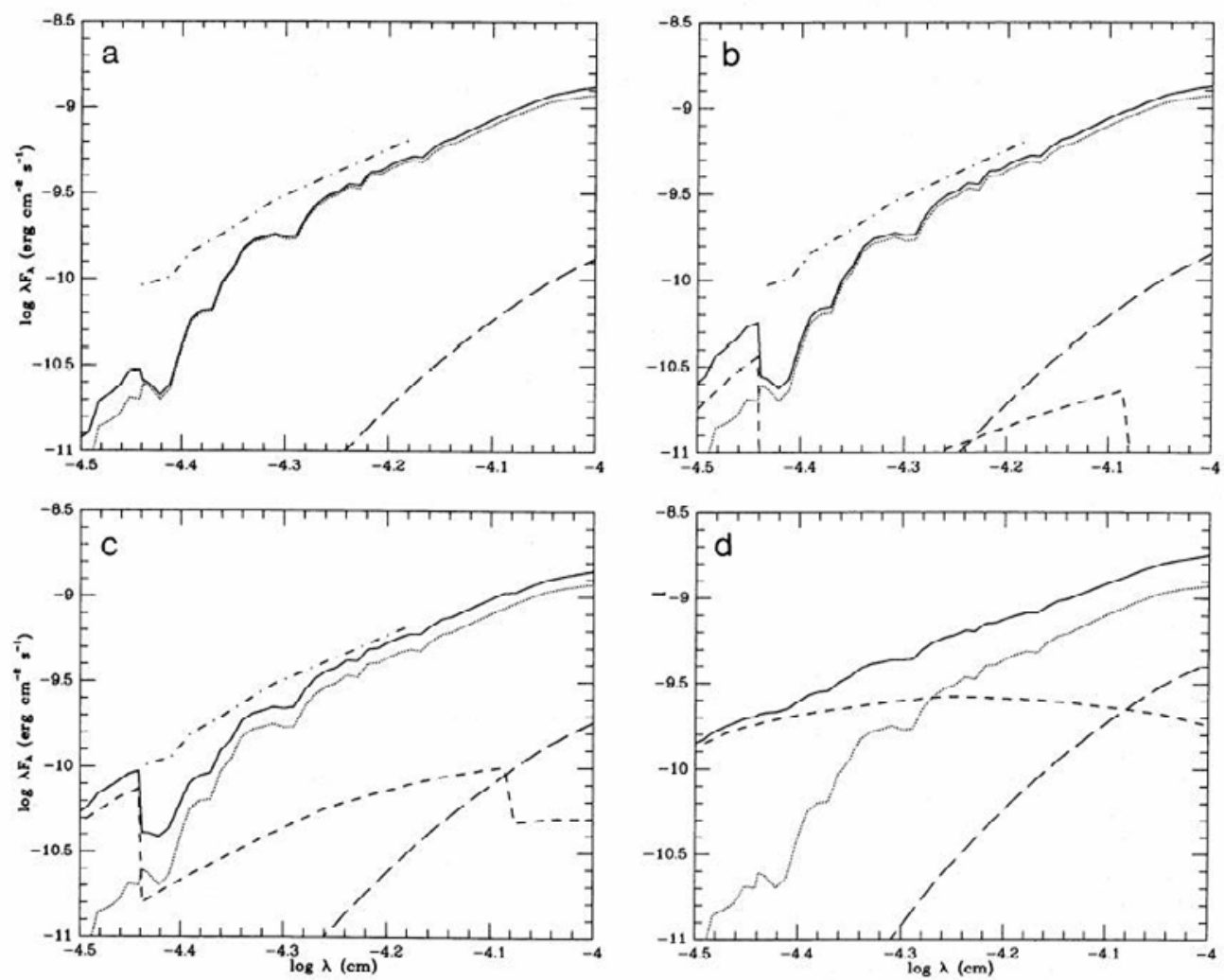

Figure 2. The veiling continuum. The spectral energy distributions arising from four combinations of a star with a disk providing increasing accretion rates from $3 \times 10^{-9}$ to $2 \times 10^{-7}$ (from Basri \& Bertout 1989). The solid line is the composite spectrum made from the star (dots) and accretion shock emission (dashed). The dash-dot line indicates where the peaks of the Balmer lines would lie if they arose only on the star.

observer. Evidence for winds is often also seen in blue-shifted absorption components in the higher Balmer series, $\mathrm{NaD}$ lines, $\mathrm{CaII} \mathrm{H} \& \mathrm{~K}$ lines, and other strong lines. A sample of $\mathrm{H} \alpha$ profiles is shown in Figure 3.

Less obvious at first, but still noted enough to create a separate subtype of young stars (the YY Ori stars; Walker 1972) is the presence of red-shifted absorption components. These are seen less often in $\mathrm{H} \alpha$ (for technical reasons due to the physical properties of the line formation region and NLTE effects), but can be seen in many of the other lines noted in the paragraph above. The same arguments there apply here but with the sign of the flow reversed - these spectral features must indicate inflow. In the early days when spherical symmetry was often invoked, it was puzzling to find signs of outflow and inflow in the same star. This was an early sign that spherical symmetry does not really apply to TTS, and could have been taken as evidence for disk accretion. When the disk accretion hypothesis began to take hold in the middle 1980s, the first conception was that the disk extended right up to the star, and accretion occurred in a "boundary layer". One of the strong pieces of evidence that magnetospheric accretion is a better picture comes from the actual infall velocities, which are closer to free-fall rather than orbital velocities (Edwards et al. 1994). A sample of $\mathrm{H} \beta$ profiles from that paper is given in Figure 4. 

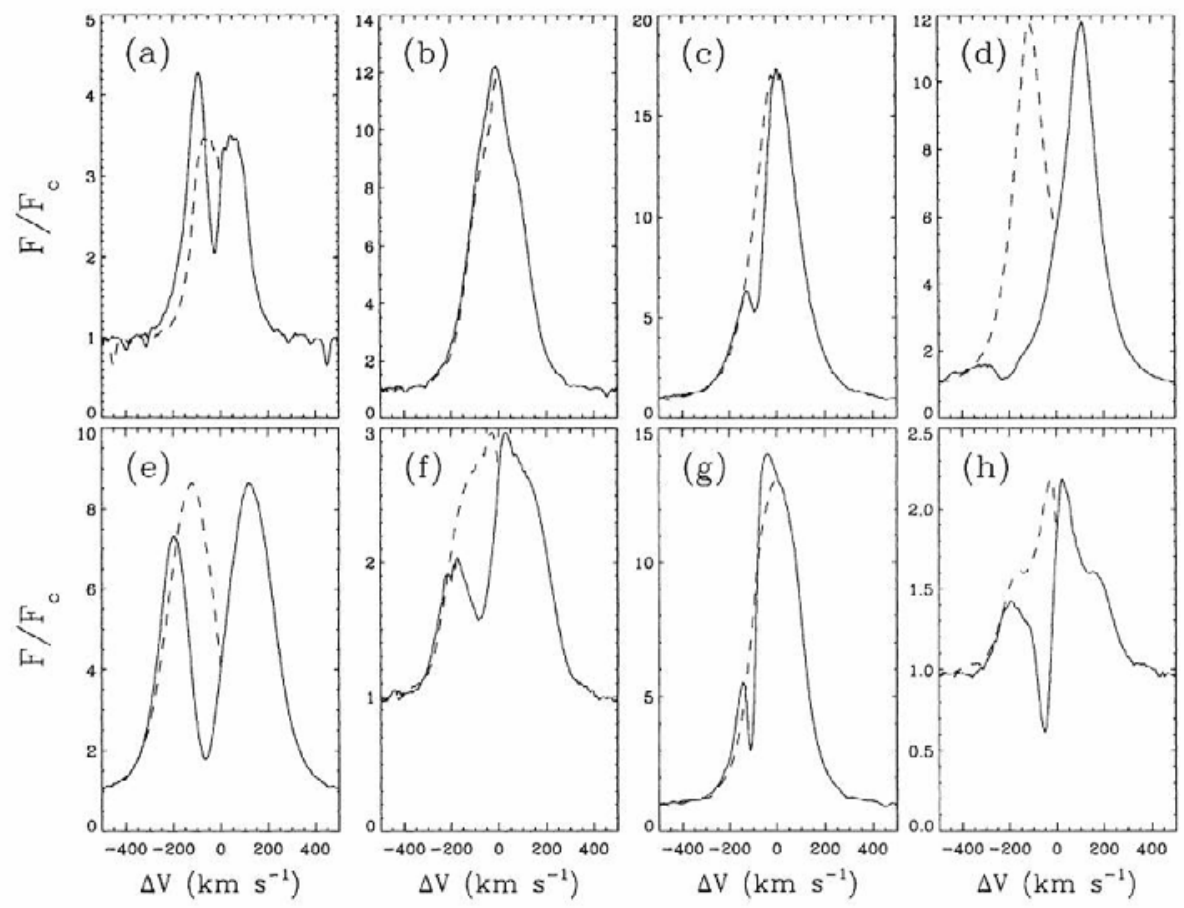

Figure 3. $\mathrm{H} \alpha$ lines in TTS. A representative set of $\mathrm{H} \alpha$ profiles taken from Johns \& Basri (1995a). Note the fact that some of the profiles have peaks far above the continuum (difficult to obtain without a large emitting volume). Superposed on the data are the red sides of the profile reflected about the stellar rest velocity (dashed lines). Note the common presence of blue-shifted absorption (though with different manifestations), and the far wings are often symmetric. The stars shown are AA Tau, BP Tau, DF Tau, DR Tau, RW Aur, RY Aur, T Tau, and SU Aur.

Beyond the obvious imprint of dynamical flows, the interpretation of strong emission line profiles in TTS has been rather vexing. The Balmer lines have always been the iconic spectral diagnostics, yet they combine the most difficult aspects of radiative transfer into a single package. They are very optically thick, arise from a high energy lower level which causes NLTE effects to play an important role, form at a temperature which typically requires non-radiative heating to play an important role, arise not only in the stelllar chromosphere but also both accretion and outflow regions which are not spherically or even axisymmetric, and are quite variable (reflecting the complexities of their formation). The result of all this is that it is difficult to properly model them from both a physical and radiative transfer point of view, and even more difficult to obtain unique models which are clearly correct.

An example of this is provided by some early work by Kuhi (1964) and Ulrich (1976). Both authors are able to plausibly reproduce the (small) sample of line profiles they are working with. Kuhi assumes a spherical outflow scenario requiring ballistic ejection from the stellar surface and ionization due to dissipation of stellar magnetic energy (making an early guess that stellar magnetic fields play a role in angular momentum losses). Ulrich assumes an infall model in which interstellar magnetic fields control the flow down to an accretion shock on the star, producing primary and secondary cooling regions which produce profiles that depend fairly strongly on their inclination to the observer. This is an early version of a magnetic accretion model, but the field is not the star's. Remarkably, this model produces profiles which also look like they have blue-shifted 

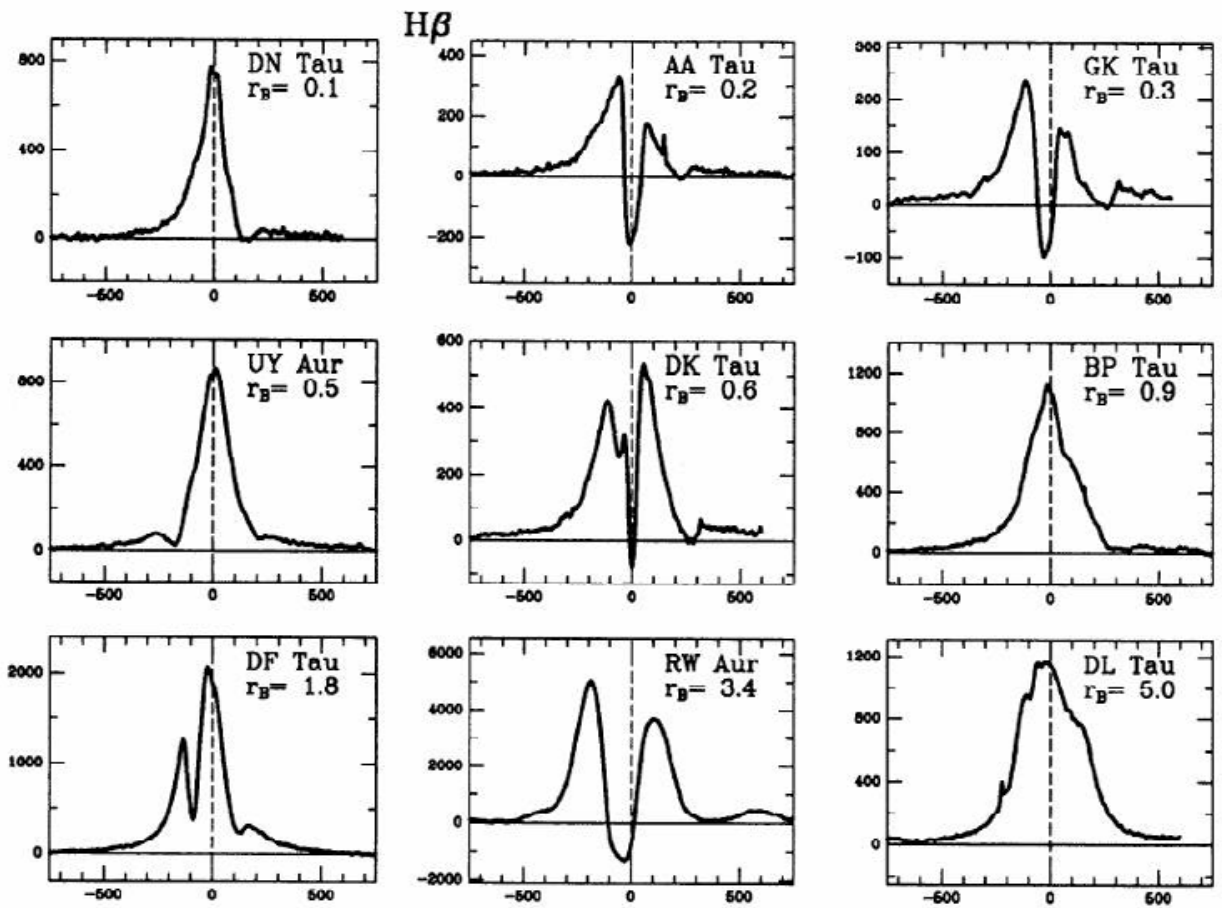

Figure 4. $\mathrm{H} \beta$ lines in TTS. A representative set of line profiles taken from Edwards et al. (1994). The vertical scale is arbitary and the horizontal scale is in $\mathrm{km} / \mathrm{s}$. The amount of blue continuum veiling (a measure of the strength of accretion) is given as a value of $\mathrm{r}_{B}$ for each spectrum.

absorption. Although neither paper passes the muster of modern spectral modeling, they are both plausible and clever efforts for their time, and still make the point that line profiles can be deceiving.

A series of other papers wrestled not only with producing profiles but also plausible physical mechanisms for producing the sort of mass loss implied by these models. That is not that easy, given that neither radiation pressure nor a thermal Parker wind is likely to work in TTS at the required level of mass loss. A suggestion of Alfven-wave driven winds remained on the table. The case for spherical outflow was finally put to rest by Hartmann et al. (1990), who showed that more careful calculations of such winds produce classical P Cygni profiles rather than the typical TTS profile. Attention then turned to the magnetospheric accretion hypothesis (see below), while it was realized that other diagnostics provided a better approach to the study of the outflow region(s). The NaD lines have always had obvious wind components (which go below the continuum, and are easier to interpret), while forbidden lines diagnose the wind (or jet) further from the star. Recently, the HeI $10830 \AA$ line (which was waiting for good detectors in that spectral region to come along) looks very promising (cf. Edwards, this volume).

\section{Diagnostics of Magnetospheric Accretion}

Once it became apparent that classical TTS are surrounded by accretion disks, thought turned to how material actually gets to the star, and how the angular momentum problem is solved (since it is clear that TTS rotate far below breakup velocities). The original idea was that the disk extended right to the star, where material lost the other half of 
its original potential energy in slowing from orbital velocity to the slow stellar surface in a boundary layer (Lynden-Bell \& Pringle 1974; Bertout, Basri \& Bouvier 1988). The difference between this and the current idea is that in magnetospheric accretion the material is slowed at several stellar radii and more or less freefalls to the stellar surface. This produces higher infall velocity signatures in line profiles, a stronger and hotter accretion shock, and moves the position of the shock from the equator to higher latitudes. Depending on the stellar field configuration, it can also produce asymmetrical shock regions (with respect to the stellar rotation axis), modulation of the accretion signature by stellar rotation, and much greater chances for short timescale variability. Indeed, Bertout, Basri \& Bouvier (1988) noted periodic variability in the blue excess from DF Tau and ascribed it to magnetically controlled accretion. One can estimate the extend of the stellar surface which is covered by shocks from the veiling; it is at most a few percent (smaller than simple dipole models predict - likely indicating a more complicated field configuration).

\subsection{Time Variability}

The variability of TTS spectra (and photometry) was one of the early defining characteristics of these objects. Examples of papers examining spectral variability at low dispersion include Aiad et al. (1984) who found variability in as little as 2 hours, and even 10 minutes at high dispersion (Mundt \& Giampapa 1982). Such short timescales are a direct indication that we are observing phenomena near the star, since it is not possible for substantial profile changes to occur over large-scale regions in such short coordinated intervals. That these changes occur in the outflow absorption features similarly argues that the part of the wind absorption region being sampled cannot arise over a very big part of the disk, and makes it more likely that these variations probe a part of outflow arising from near the magnetospheric boundary. Even more direct evidence of magnetospheric influence can come from the modulation of brightness or profile changes which have the stellar rotation period.

One of the clearest examples of a profile variability study showing that there are 3 components of the $\mathrm{H} \alpha$ profile which arise from the stellar chromosphere, magnetospheric accretion, and an outflow modulated by the stellar field is the study of SU Aur by Giampapa et al. (1993) and Johns \& Basri (1995b). A collection of more than 100 high resolution spectra found clear periodicity consistent with the stellar rotation period in both infall (in $\mathrm{H} \beta$ ) and outflow profile components (in $\mathrm{H} \alpha$ and $\mathrm{H} \beta$ ) as shown in Figure 5 . Even more interestingly, these components were out of phase: one side of the system shows stronger inflow and the other side stronger outflow. They are interpreted as caused by a tilted dipole field (with respect to the stellar rotation axis and the disk plane), and the connection with stellar rotation leaves little room for alternatives to the stellar magnetic field as the source of the variations.

These papers also introduced two ways of displaying the information inherent in time series of high resolution profiles. One is to simply display the normalized variance profile along with the average profile, which highlights the velocities at which the profile displays most of its variability. This typically shows that the most variability is associated with the outflow part of the profile, even when an absorption feature is not very strong. It also highlights the infall variability, showing red-shifted variance features in $\mathrm{H} \alpha$ even when there is no overt absorption at all. To study infall, however, it is better to concentrate on $\mathrm{H} \beta$ (Edwards et al. 1994). That paper made it clear that the infall velocities are high enough that magnetospheric accretion is the preferred interpretation, particularly since the strength of the absorption is correlated with other accretion diagnostics like veiling. 


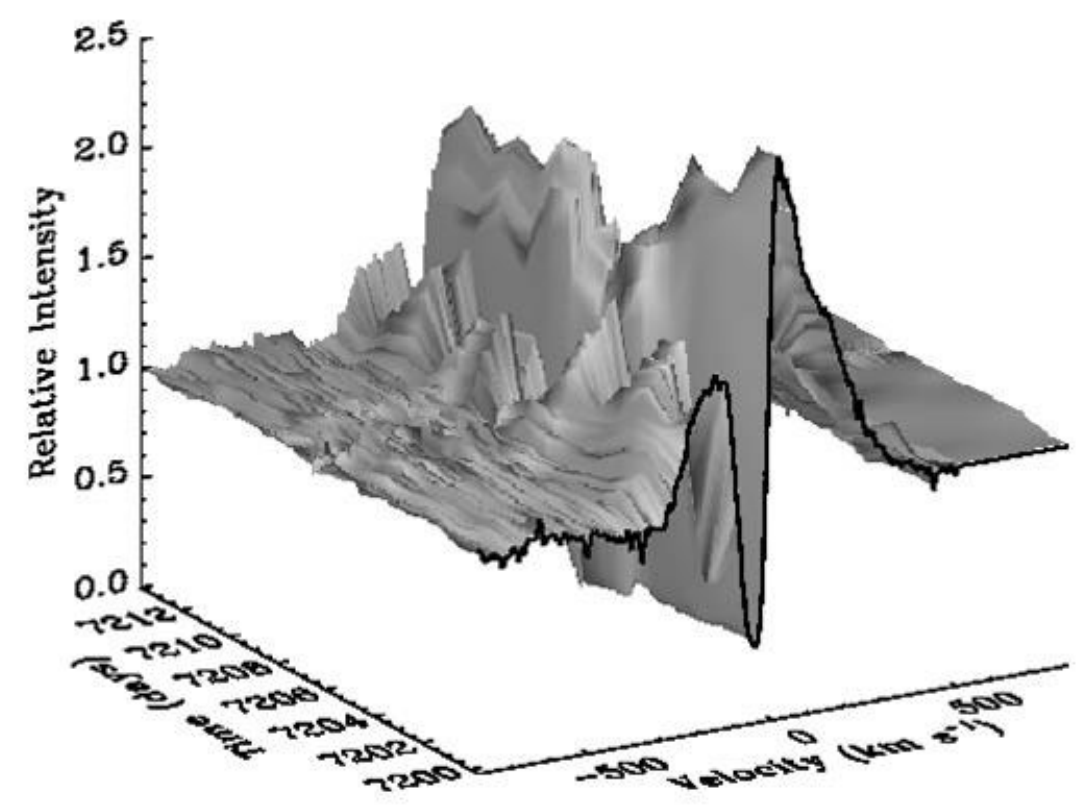

Figure 5. Profile variability in SU Aur. A time-resolved history of the $\mathrm{H} \alpha$ profile in SU Aur. Notice the periodic variation of the blue side of the line, showing an outflow component that is modulated by the stellar magnetic field on the stellar rotation period.

The other means of studying profile variability is using "correlograms" (Johns \& Basri 1995a), which show the extent to which variations at any velocity in the profile are correlated with variations at any other velocity. If the variations of different parts of the profile are uncorrelated, one gets a narrow diagonal line whose width shows the extent in velocity of the locality (probably induced by local turbulence). When the whole profile moves together, the correlogram takes on a blocky or square appearance. Both sorts of behavior are observed on TTS, and the use of this sort of diagnostic is just coming into play from a modeling point of view (Kurosawa, Harries \& Symington 2006).

\subsection{Profile Modeling}

The modeling of the actual profiles seen in TTS is one of the hardest tasks facing us today. As mentioned at the beginning, there are many reasons for this because of the inherent geometrical complexity of the emitting region, our ignorance of the physical conditions in it (velocities, densities, and heating rates), and details of radiative transfer. A series of papers by Muzerolle and collaborators (Muzerolle, Calvet \& Hartmann 1998, 2001; and other papers for higher and lower mass objects) constitutes the primary standard for such work. They use "cartoon" models for the geometry and physical conditions, but a reasonable (although still axisymmetric) approach to the radiative transfer. These calculations show that the magnetospheric hypothesis produces plausible line profiles (Figure 6), and allows estimation of accretion rates from high resolution spectra. The extent to which actual TTS resemble these computed profiles has been discussed by the CfA group who note the areas of agreement, and by Alencar \& Basri (2000) who also point out the substantial exceptions. Since these models are quite physically simplistic, 

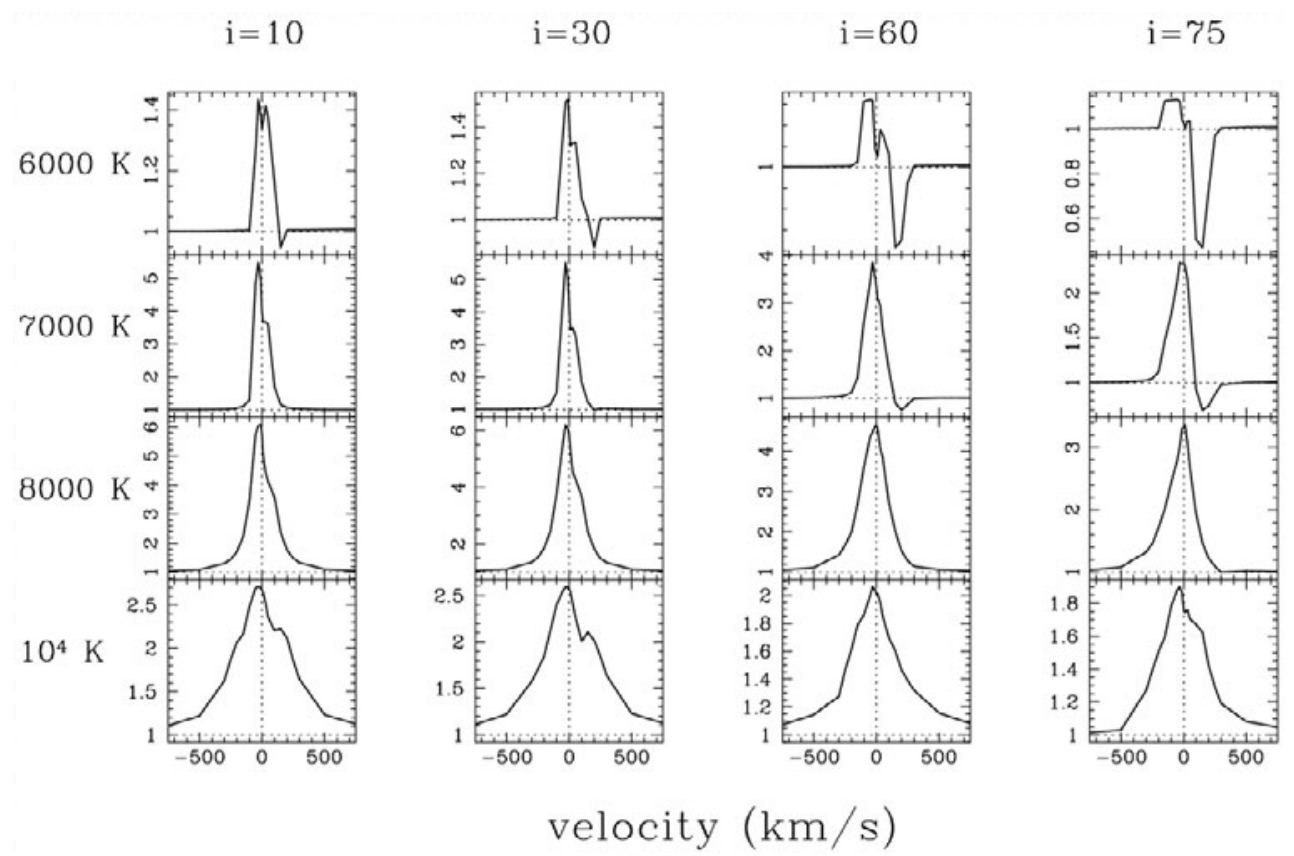

Figure 6. Computed magnetospheric profiles. A set of $\mathrm{H} \beta$ profiles from the work of Muzerolle, Calvet \& Hartmann (2001). Here the effect of different amounts of heating viewed at different inclinations are shown. The infall absorption feature is more obvious at high inclination, the magnetosphere produces a broader profile if it is hotter, and there is a characteristic asymmetry in the emission profile which is clearer for cooler, more edge-on cases.

the extent of agreement is quite heartening, and there is general consensus that this is the correct paradigm to use.

More recently, there has been an explosion of more sophisticated efforts which use more physically realistic modeling. These compute more realistic magnetic configurations, use good radiative transfer, and work on variability as well. They are the subject of a number of papers in this volume, and I commend the reader to them. The Balmer lines are the most difficult case, although they also span the greatest physical region. It will be important for the future to examine other diagnostics in greater detail, being sure to conduct synoptic observations so that the range of variability and the appearance of a given system at different phases are included in our understanding of these highly complex and ever-changing systems. The reward is a detailed understanding of the processes which are at the basis of the near stellar environment during star formation, and which tell us how the star manages to collect material and dump angular momentum to grow into the final product.

\section{Acknowledgements}

I would like to acknowledge the enjoyable and productive collaborations and discussions I have had with Claude Bertout and his students and collaborators over the past 2 decades. These have made my sojourn in the area of star formation much more enjoyable and effective. I would also like to thank my students: Jeff Valenti, Chris Johns-Krull, Subu Mohanty, and David Ardila for choosing to work with me and then making me very proud to have worked with them. I also acknowledge the ongoing support of the National Science Foundation through a series of grants. 


\section{References}

Alencar, S.H.P., Basri, G. 2000 AJ 119, 1881

Aiad, A., Appenzeller, I., Bertout, C., Stahl, O., Wolf, B., Isobe, S., Shimizu, M., Walker, M. F. 1984 A\&A 130, 67

Basri, G. \& Bertout, C. 1989 ApJ 341, 340

Basri, G., Batalha, C. 1990 ApJ 363, 654

Basri, G., Marcy, G.M. \& Valenti, J. 1992 ApJ 390, 622

Bertout, C. 1989 ARAA 27, 351

Bertout, C., Basri, G. \& Bouvier, J. 1988 ApJ 330, 350

Calvet, N., Basri, G. \& Kuhi, L.V. 1984 ApJ 277, 725

Calvet, N., Basri, G., Imhoff, C., Giampapa, M. 1985 ApJ 293, 575

Dumont, S., Heidmann, N., Kuhi, L. V., Thomas, R. N. 1973 A $\& A$ 29, 199

Edwards, S., Hartigan, P., Ghandour, L., Andrulis, C. 1994 AJ 108, 1056

Finkenzeller, U., Basri, G. 1987 ApJ 318, 823

Giampapa, M.S., Basri, G.S., Johns, C.M., Imhoff, C. 1993 ApJS 89, 321

Gullbring, E., Hartmann, L., Briceño, C., Calvet, N. 1998 ApJ 492, 323

Hartmann, L., Avrett, E.H., Loeser, R., Calvet, N. 1990 ApJ 349, 168

Hartigan, P., Hartmann, L., Kenyon, S., Hewett, R., Stauffer, J. 1989 ApJS 70, 899

Hartigan, P., Edwards, S. \& Ghandour, L. 1995 ApJ 452, 736

Herbig, G.H. 1985 ApJ 289, 269

Johns, C.M., Basri, G. 1995a $A J$ 109, 2800

Johns, C.M., Basri, G. 1995b ApJ 449, 341

Joy, A.H. 1945 ApJ 102, 168

Kurosawa, R., Harries, T.J. \& Symington, N.H. 2006 MNRAS 370, 580

Kuhi, L.V. 1964 ApJ 102, 168

Lynden-Bell, D., Pringle, J.E. 1974 MNRAS 168, 603

Mundt, R., Giampapa, M.S. 1982 ApJ 256, 156

Muzerolle, J., Calvet, N. \& Hartmann, L. 1998 ApJ 492, 743

Muzerolle, J., Calvet, N. \& Hartmann, L. 2001 ApJ 550, 944

Rydgren, A.E., Strom, S.E. \& Strom, K.M. 1976 ApJS 30, 307

Ulrich, R. 1976 ApJ 210, 377

Valenti, J., Basri, G. \& Johns, C.M. 1993 AJ 106, 2024

Walker, M.F. 1972 ApJ 175, 89

White, R., Basri, G. 2003 ApJ 582, 1109

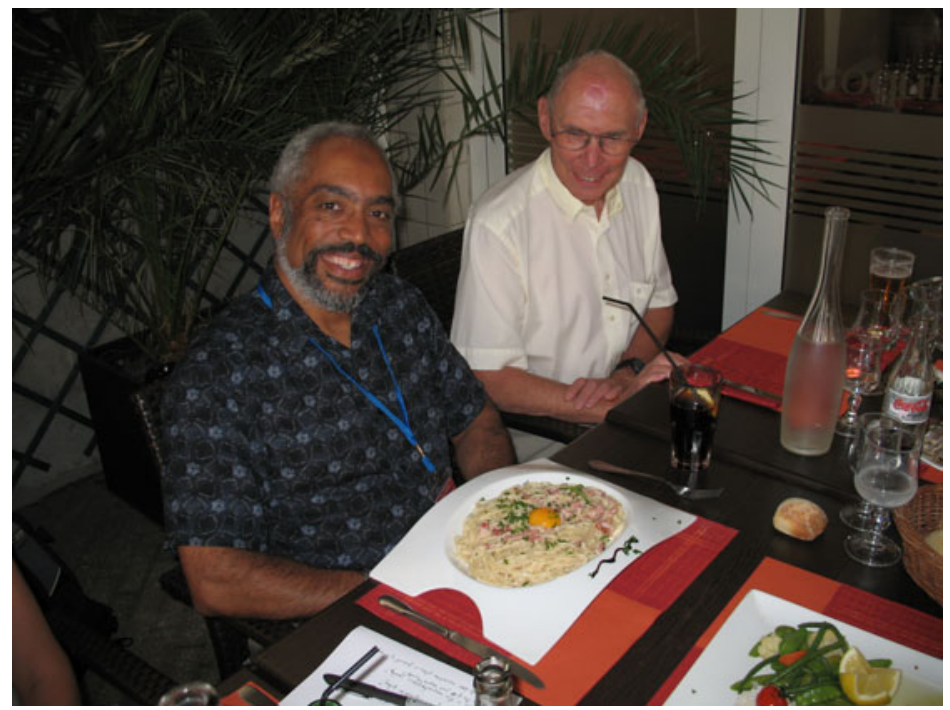

